\title{
ESTUDIO DEL COMPORTAMIENTO DE MORTEROS CEMENTO-ARENA EN PROPORCIONES 1:3 Y 1:4 PARA DIFERENTES PORCIENTOS DE SUSTITUCIÓN DE ARENA NATURAL POR RECICLADA
}

\author{
Study of the behavior of mortar sands in 1:3 and 1:4 proportions for \\ different percents of substitution of natural sand by recycling
}

\section{EPISTEMUS}

ISSN: 2007-8196 (electrónico)

ISSN: 2007-4530 (impresa)

Israel Miranda Pasos ${ }^{1}$

Jesús Quintana Pacheco ${ }^{2}$

Jesús Fernando García Arvizu ${ }^{3}$

Samuel Castro Brockman ${ }^{4}$

Recibido: 27 de noviembre de 2017

Aceptado: 22 de mayo de 2018

Autor de Correspondencia:

Dr. Israel Miranda Pasos

Correo: israel.miranda@unison.mx

\section{Resumen}

El proyecto evaluó el comportamiento del mortero hidráulico en las proporciones 1:3 y 1:4, en estado fresco y endurecido, al sustituir el agregado natural por agregado reciclado en los porcentajes del $10 \%, 20 \%$, $30 \% 40 \%$ y $50 \%$. La evaluación se enfoca en la resistencia a compresión simple, estado fresco: la masa unitaria y la fluidez. Tomando en cuenta los resultados obtenidos en ambos morteros, la resistencia en la proporción 1:4 fue aumentando para todos los porcientos de sustitución con respecto al mortero base 1:4. En el caso del mortero de proporción 1:3 el mejor comportamiento se obtuvo con la sustitución del $20 \%$ con respecto al base 1:3. La demanda de agua se incrementó para las dos proporciones en sus diferentes por cientos de sustitución de agregado reciclado, éste incremento obedece al mayor por ciento de absorción que tiene el agregado reciclado con respecto al natural.

Palabras claves: Mortero hidráulico, Agregado reciclado, Porcentaje sustitución, Resistencia, Fluidez.

\section{Abstract}

The project evaluated the behavior of the hydraulic mortar in the proportions 1: 3 and 1: 4, in a fresh and hardened condition, replacing the natural aggregate by recycled aggregate in the percentages of 10\%, 20\%, 30\%, $40 \%$, and $50 \%$. The evaluation focuses on resistance to simple compression, freshness; unit mass and fluidity. Taking into account the results obtained in both mortars, the resistance in the ratio 1: 4 was increasing for all the percentages of substitution with respect to the mortar base 1: 4 . In the case of mortar of ratio 1: 3 the best performance was obtained with the substitution of 20\% with respect to the base 1:3. The demand for water increased for both proportions in its different percentages of substitution of recycled aggregate, this increase is due to the greater percentage of absorption that the recycled aggregate has with respect to the natural one.

Keywords: Hydraulic mortar, Recycled aggregate, Substitution percentage, Resistance, Fluidity.

1 Universidad de Sonora, México / Correo: imiranda@dicym.uson.mx 2 Universidad de Sonora, México / Correo: quintana@dicym.uson.mx. 3 Universidad de Sonora, México / Correo: fergarcia@pitic.uson.mx.

4 Universidad de Sonora, México / Correo: sam2032@gmail.com 


\section{INTRODUCCIÓN}

Los residuos sólidos municipales (RSM) urbanos provienen de diferentes fuentes de generación como; las viviendas, los comercios, las tiendas de conveniencia, los supermercados, los parques, la construcción de obra civil, entre otras, siendo éste último el sector el que genere una importante cantidad de residuos de manejo especial, en especial los escombros producto de la demolición y/o deconstrucción.

En la ciudad de Hermosillo, Sonora, los residuos de construcción, empieza a ser un serio problema, por lo que el presente proyecto evaluó el comportamiento del mortero hidráulico en la proporciones $1: 3$ y 1:4, en estado fresco y endurecido, al sustituir el agregado natural por agregado reciclado en los porcentajes del 10\%, 20\%, 30\% $40 \%$ y $50 \%$. Se enfocó a la evaluación de la resistencia a compresión simple, así como la masa unitaria y la fluidez.

Tomando en cuenta los resultados en la presente investigación, la resistencia del mortero elaborado con sustitución parcial de agregado fue mayor para todos los porcientos de sustitución con respecto a la base.

Las propiedades en estado fresco; la masa unitaria y la fluidez, la primera se observa que disminuye conforme aumenta el contenido de agregado reciclado $y$, para mantener la fluidez en el rango, la demanda de agua aumenta conforme aumenta el porcentaje de sustitución.

\section{Sitio de estudio}

El presente trabajo se desarrollara en dos etapas: la primera etapa correspondiente al proceso de reciclaje (trituración y cribado), se llevó a cabo con equipos de trituración propiedad de la Universidad de Sonora y ubicados en el laboratorio experimental de ingeniería civil, del departamento de ingeniería civil y minas. La segunda etapa correspondiente a los ensayos y pruebas de los materiales, se realizó en las instalaciones

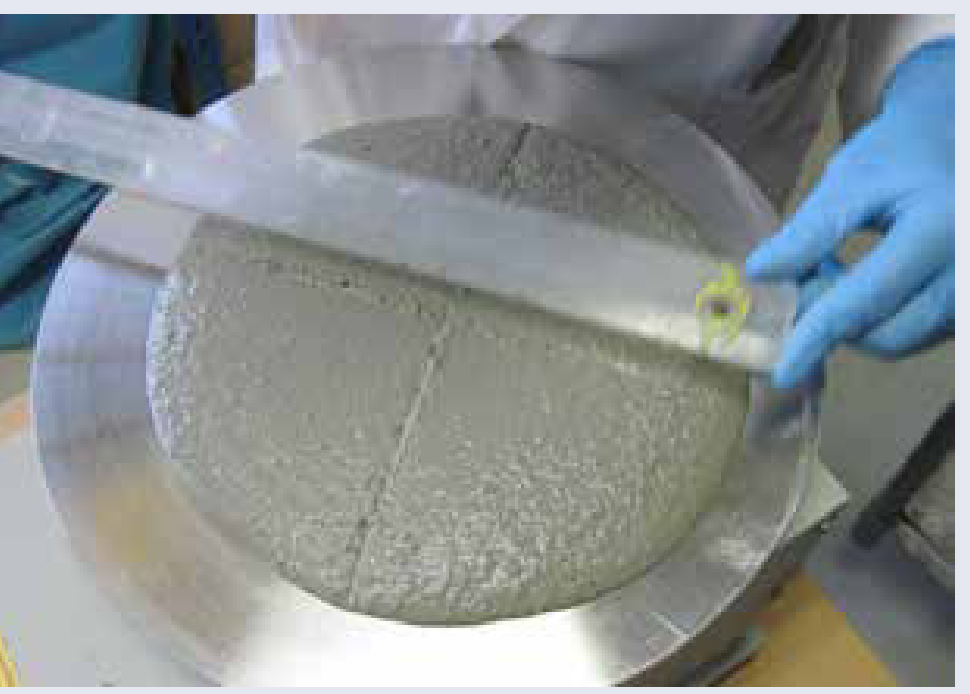

de Laboratorio Experimental de Ingeniería, del Departamento de Ingeniería Civil de la Universidad de Sonora, Unidad regional Centro.

\section{MATERIALES Y MÉTODOS \\ Materiales y equipos}

Apegándose a criterios de muestreo, se colectaron la mayor cantidad posible de escombro de concreto producto de la demolición, los cuales forman parte de la materia prima, para poder llevar a cabo el proceso de trituración y de ellos obtener el agregado con el cual se elaboró el mortero, éste fue sometido a un procesos de trituración, trituradora de quijada y de cono, para obtener la granulometría necesaria con calidad arena.

Respecto al agregado natural (arenas) este corresponde al obtenido de los bancos en servicio para el sector de la construcción, como lo es el Banco "Castillo", localizado a las márgenes del Río Sonora, al sur poniente de la mancha urbana sobre el Río.

Para los ensayos y pruebas en laboratorio se usó la prensa universal; para la compresión y tensión, balanzas, hornos electrónicos, mesa de fluidez, así como las diferentes mallas, termómetro y equipo de laboratorio menor.

\section{Muestreo de la materia prima.}

Se recolectó la mayor cantidad de residuos de concreto. La carga del material al vehículo se hizo de manera manual, con la finalidad de separar las impurezas del escombro del concreto, es decir, verificando que la materia prima esté libre de materiales como: materia orgánica, plásticos, papeles, vidrios, madera y acero.

\section{Obtención y caracterización de agregados.}

El agregado fino reciclado se obtiene mediante la trituración del concreto producto de la demolición de obras civiles y recolectadas en escombreras. El agregado fino natural se adquirirá del banco de materiales "Castillo".

Una vez obtenido el agregado fino natural y el producto de la trituración, se trasladaron al laboratorio con el fin de someterlos a los ensayos correspondientes para determinar su caracterización física.

\section{Caracterización}

Primeramente se estudiaron los agregados para la obtención de las propiedades de: Distribución granulométrica (NMX-C-077- ONNCCE-1997), Masa específica y absorción gravas (NMX-C-164ONNCCE-2002), masa específica y absorción arenas (NMX-C-165-ONNCCE-2004), masa volumétrica (NMXC-073-ONNCCE-2004), humedad natural (NMX-C-166ONNCCE-2006), resistencia a la abrasión (NMX-C-196ONNCCE-2010) y contenido de finos $(<0.075 \mathrm{~mm}$ ) ( NMX-C-084-ONNCCE 2002). 


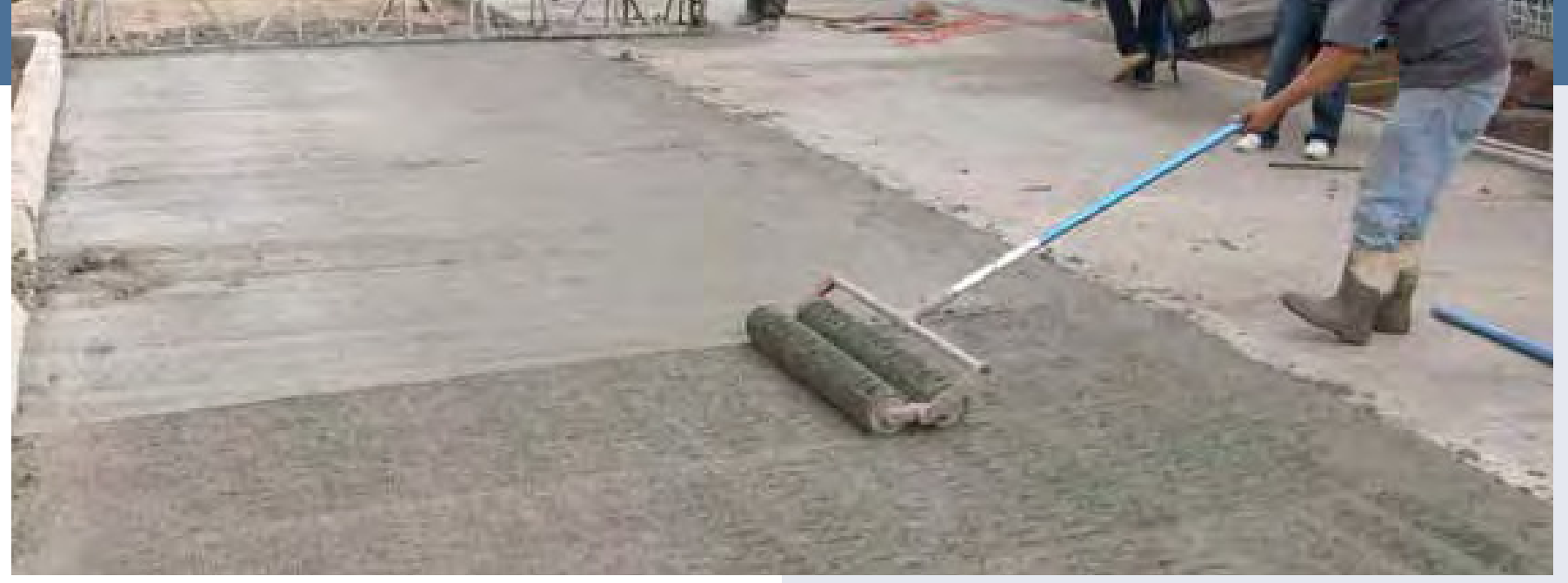

\section{Diseño y elaboración de mortero.}

Buscando cumplir con las características deseables para un mortero, principalmente resistencia y durabilidad. Esto de acuerdo a los procedimientos de diseño de mezclas del ACl 211 (American Concrete Institute), (imcyc,20014). El mortero se diseñó para una relación 1:4 y 1:3. La primera corresponde a la fabricación de mortero ordinario para muros de carga y la segunda para morteros resistentes (ricos) para enlucidos, impermeabilizaciones, revoques, afine de pisos, impermeabilizaciones, entre otras.

\section{Procedimiento para mezclar morteros}

Se colocan la olla y la paleta secas en la mezcladora, en posición de mezclado. Se vierten los materiales necesarios para la cantidad estipulada de mezcla en la olla, efectuando las operaciones de la forma siguiente:

$\diamond$ Se vierte el agua dentro de la olla

$\diamond$ Se agrega el cemento a la olla y se hace funcionar la mezcladora en velocidad baja ( $140 \mathrm{r} / \mathrm{min}$ :t $5 \mathrm{r} / \mathrm{min}$ ) durante $30 \mathrm{~s}$

$\diamond$ Se agrega la cantidad total de arena vertiéndola lentamente al mismo tiempo que la mezcladora este funcionando a la velocidad baja durante $30 \mathrm{~s}$.

$\diamond$ Se detiene la mezcladora y cambiando a la velocidad alta a $(285 \mathrm{r} / \mathrm{min}$ :t $10 \mathrm{r} / \mathrm{min})$ se hace funcionar la mezcladora durante $30 \mathrm{~s}$.

$\diamond$ Se detiene la mezcladora y se deja reposar la mezcla durante 1,5 min; durante los primeros $15 \mathrm{~s}$ de este tiempo se desprende rápidamente hacia abajo con la espátula todo el mortero que se haya adherido a las paredes de la olla, inmediatamente se cubre la olla con la tapa hasta que se complete el minuto y medio. Se termina el mezclado haciendo funcionar la mezcladora durante un minuto más a la velocidad alta (285 r/min:t $10 \mathrm{r} / \mathrm{min})$.

En cualquiera de las ocasiones en las que se efectúe una operación de remezclado se debe desprender rápidamente hacia abajo, por medio de la espátula, todo el mortero adherido a las paredes de la olla, antes de comenzar el remezclado.

\section{Pruebas de laboratorio}

Los ensayes a los morteros serán en estado fresco y endurecido, en estado freso los ensayes son:

- Fluidez.- Este ensaye consiste el llenado de un molde tronco cónico, el cual se coloca en el centro del plato, se vierte una capa del mortero que se requiere ensayar. El molde se llena en dos capas de 1" de espesor aproximadamente, se apisona 20 vece, uniformemente distribuidos, se coloca la segunda capa y se apisona de la misma forma que la primera y se enrasa. La presión del compactador, será la suficiente de tal manera que asegure el llenado uniforme del molde.

Una vez enrasado el mortero en el molde, se limpia el plato, teniendo cuidado de secar el agua alrededor del molde. Después de transcurrido 1 minuto contado a partir del enrase, se retira el molde e inmediatamente se inicia con el proceso de dejar caer la mesa 25 veces una altura de $12.7 \mathrm{~mm}$ en 15 segundos. Luego se mide el diámetro de la base de la muestra que se extendió en cuatro puntos equidistantes y se calcula el diámetro promedio. (ICG, 2013 y NMX-C-085-ONNCCE-2002).

La fluidez es el aumento del diámetro de la muestra expresado en por ciento del diámetro con respecto al diámetro base (diámetro de la base del cono truncado). La fórmula para su cálculo es:

$\%$ Fluidez $=\frac{\text { Diámetro promedio en } \mathrm{mm}-101.6 \mathrm{~mm}}{101.6 \mathrm{~mm}} \times 100$

Masa volumétrica.- Corresponde a la cantidad de masa contenida en un volumen determinado.

En estado endurecido son:

- Resistencia a la Compresión.- Es la capacidad de resistir esfuerzos a compresión de un elemento elaborado a base de cemento-arena y agua. Para morteros se aplica la norma NMX-C-061ONNCCE-2001. 


\section{Secuencia del proceso experimental de laboratorio}

\section{Preparación de materiales.-}

Las materiales obtenidos producto de la trituración se caracterizarán física y mecánicamente para su clasificación.

$>\quad$ Diseño de mezclas.- Se realizará el diseño de mezclas para mortero hidráulico a base de cemento arena; para agregado normal y reciclado, respectivamente.

Los resultados de los morteros elaborados a base de Cem- Arena (Natural-reciclada) en proporción 1:3 y 1:4 para las diferentes edades se elaboraron con arena natural y arena reciclada. La cantidad de sustitución de arena natural por reciclada fue en los porcentajes del $0 \% 10 \%, 20 \%$, $30 \%, 40 \%$ y $50 \%$ calculado en volumen absoluto.

$>$ Determinación de la resistencia.- Se elaboraron elementos prismáticos de $5 \mathrm{~cm} \times 5 \mathrm{~cm} \times 5 \mathrm{~cm}$ para obtener la resistencia a compresión. Al mortero en estado fresco se le determino la fluidez y masa volumétrica y, al mortero endurecido se determinó su resistencia a compresión simple.

\section{RESULTADOS}

\section{Resultados de la caracterización de los agregados}

La tabla1 presenta los resultados de la caracterización de los agregados finos, arena natural y arena obtenida de escombros de concreto - arena reciclada. Cabe señalar que cumplen con los límites establecidos en la normatividad mexicana, NMX.

Tabla 1.- Resultados de la caracterización del agregado fino, natural y reciclado.

\begin{tabular}{|l|c|c|c|c|c|c|}
\hline \multicolumn{1}{|c|}{ ARENA } & $\begin{array}{c}\text { DENSIDAD } \\
\mathbf{k g} / \mathbf{m}^{3}\end{array}$ & $\begin{array}{c}\text { PVSV } \\
\mathbf{k g} / \mathbf{m}^{3}\end{array}$ & $\begin{array}{c}\text { PVSS } \\
\mathbf{k g} / \mathbf{m}^{3}\end{array}$ & $\begin{array}{c}\text { ABSORCIÓN } \\
\%\end{array}$ & $\begin{array}{c}\text { HUMEDAD } \\
\text { NATURAL \% }\end{array}$ & $\mathbf{M . F}$ \\
\hline Natural & 2557 & 1665 & 1605 & 2.5 & 1.0 & 2.74 \\
\hline Reciclada & 2364 & 1403 & 1336 & 8.0 & 2.0 & 2.58 \\
\hline
\end{tabular}

La tabla 2 y 3 presenta los resúmenes de los diseños para el mortero cemento - arena 1:4 y 1:3 para los diferentes por cientos de sustitución, del 0 $\%$ hasta el $50 \%$.

Tabla 2.- Resumen de las proporciones de los diseños de mezcla mortero 1:4

\begin{tabular}{|c|c|c|c|c|c|c|}
\hline \multirow{2}{*}{ MATERIAL } & \multicolumn{6}{|c|}{$\begin{array}{l}\text { MEZCLAS TEÓRICA DE MORTERO 1:4 CON SUSTITUCIÓN PARCIAL DE ARENA } \\
\text { NATURAL POR RECICLADA. }\end{array}$} \\
\hline & $\begin{array}{c}\text { Sustitución } \\
0 \%\end{array}$ & $\begin{array}{c}\text { Sustitución } \\
10 \%\end{array}$ & $\begin{array}{c}\text { Sustitución } \\
20 \%\end{array}$ & $\begin{array}{l}\text { Sustitución } \\
30 \%\end{array}$ & $\begin{array}{l}\text { Sustitución } \\
\quad 40 \%\end{array}$ & $\begin{array}{l}\text { Sustitución } \\
\quad 50 \%\end{array}$ \\
\hline Cemento kg & 405 & 405 & 405 & 405 & 405 & 405 \\
\hline $\begin{array}{l}\text { Arena } \\
\text { Natural kg }\end{array}$ & 1382 & 1244 & 1106 & 968 & 829 & 691 \\
\hline $\begin{array}{l}\text { Arena } \\
\text { Reciclada kg }\end{array}$ & 0 & 138 & 276 & 415 & 553 & 691 \\
\hline Agua inicial (I) & 331 & 336 & 342 & 347 & 353 & 359 \\
\hline $\begin{array}{l}\text { Masa Unitaria } \\
\text { Teórica } \mathbf{k g} / \mathrm{m}^{3}\end{array}$ & 2118 & 2123 & 2129 & 2135 & 2140 & 2146 \\
\hline
\end{tabular}


Tabla 3.- Resumen de las proporciones de los diseños de mezcla mortero 1:3

\begin{tabular}{|c|c|c|c|c|c|c|}
\hline \multirow{2}{*}{ MATERIAL } & \multicolumn{6}{|c|}{$\begin{array}{l}\text { MEZCLAS TEÓRICA DE MORTERO 1:3 CON SUSTITUCIÓN } \\
\text { PARCIAL DE ARENA NATURAL POR RECICLADA }\end{array}$} \\
\hline & $\begin{array}{c}\text { Sustitución } \\
0 \%\end{array}$ & $\begin{array}{c}\text { Sustitución } \\
10 \%\end{array}$ & $\begin{array}{c}\text { Sustitución } \\
20 \%\end{array}$ & $\begin{array}{c}\text { Sustitución } \\
30 \%\end{array}$ & $\begin{array}{c}\text { Sustitución } \\
40 \%\end{array}$ & $\begin{array}{l}\text { Sustitución } \\
\mathbf{5 0 \%}\end{array}$ \\
\hline Cemento kg & 494 & 494 & 494 & 494 & 494 & 494 \\
\hline $\begin{array}{l}\text { Arena } \\
\text { Natural kg }\end{array}$ & 1266 & 1139 & 1013 & 886 & 760 & 633 \\
\hline $\begin{array}{l}\text { Arena } \\
\text { Reciclada kg }\end{array}$ & 0 & 127 & 253 & 380 & 506 & 633 \\
\hline Agua inicial (I) & 321 & 326.6 & 332.4 & 338.1 & 343.8 & 349.5 \\
\hline $\begin{array}{l}\text { Masa Unitaria } \\
\text { Teórica kg/m }\end{array}$ & 2081 & 2087 & 2092 & 2098 & 2104 & 2110 \\
\hline
\end{tabular}

\section{Resultados de compresión simple, demanda de agua y masa unitaria.-}

Éste se basa en los resultados de las pruebas y/o ensayes al mortero normal y al reciclado, tomando en cuenta las propiedades y comportamiento de los agregados utilizados.

A continuación se presentan los análisis de los resultados a través de gráficos, los cuales presentan el comportamiento de las mezclas, así como los modelos lineales (ecuaciones) que predicen de manera numérica el comportamiento de la mezcla en cuestión.

\section{Análisis de resultados}

Resultados de resistencia a compresión simple 1:4

La resistencia a compresión es mayor en el mortero elaborado con agregado reciclado para los diferentes por cientos de sustitución, como se observa en la figura 2. En la figura 1 se observa que el mortero con $0 \%$ se mantuvo por debajo para las diferentes edades del mortero.
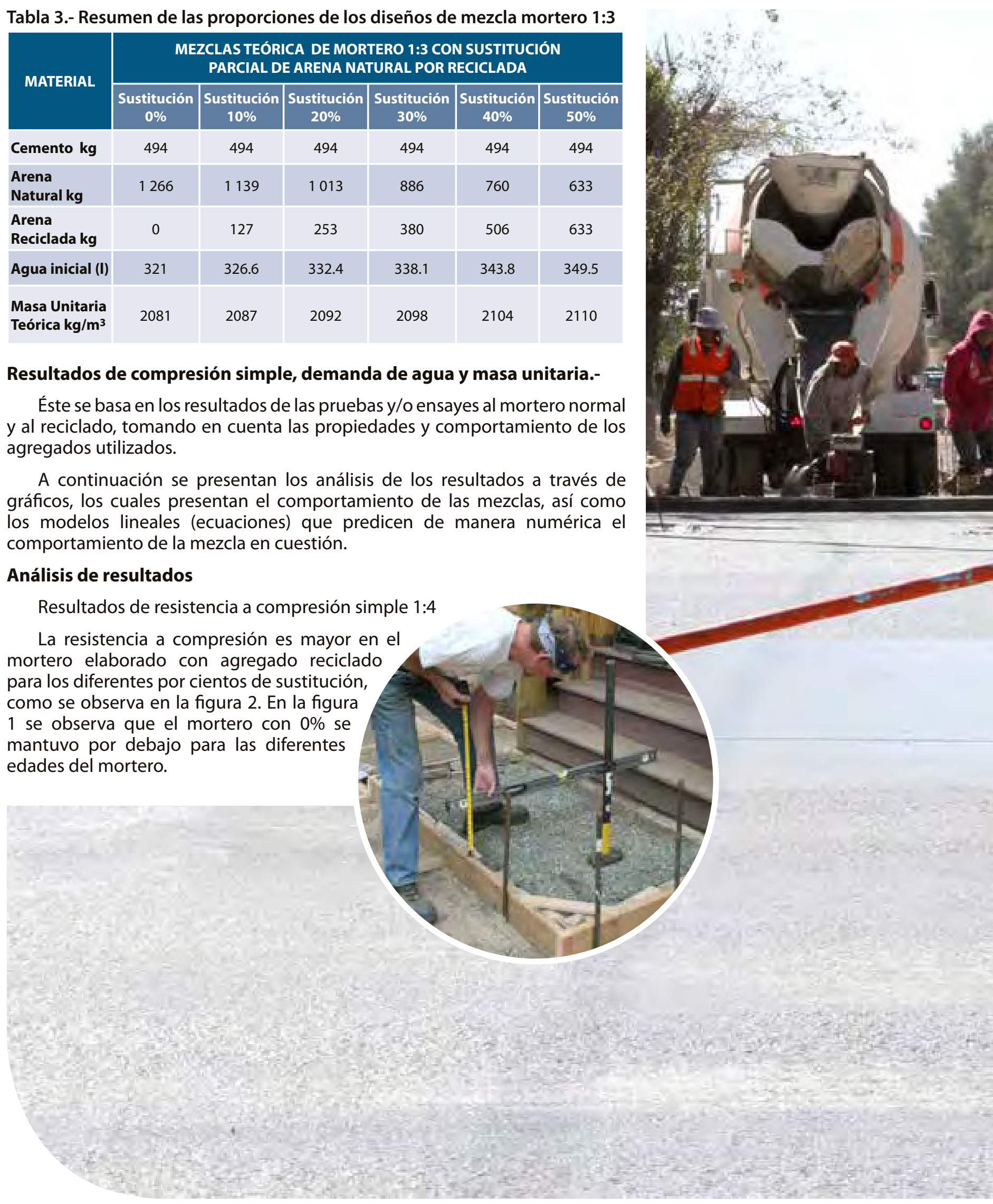


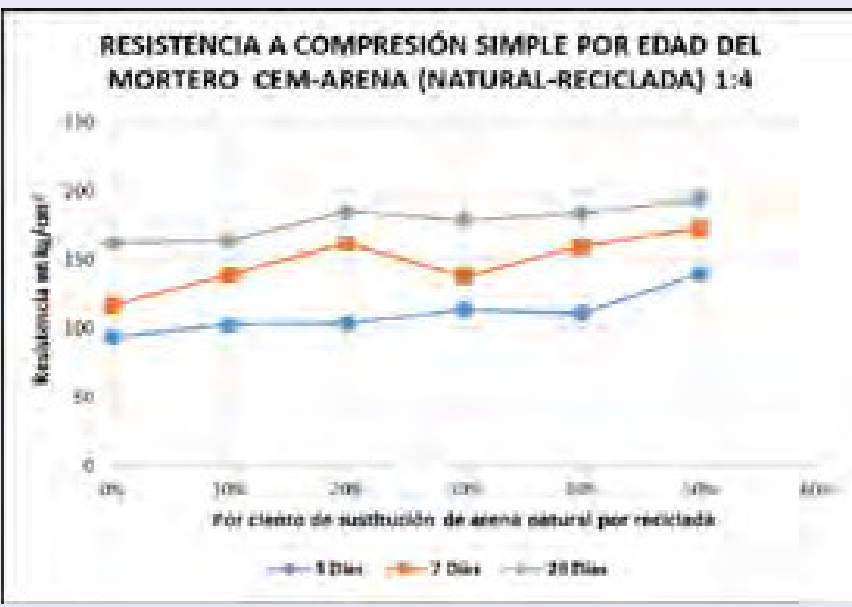

Figura 1

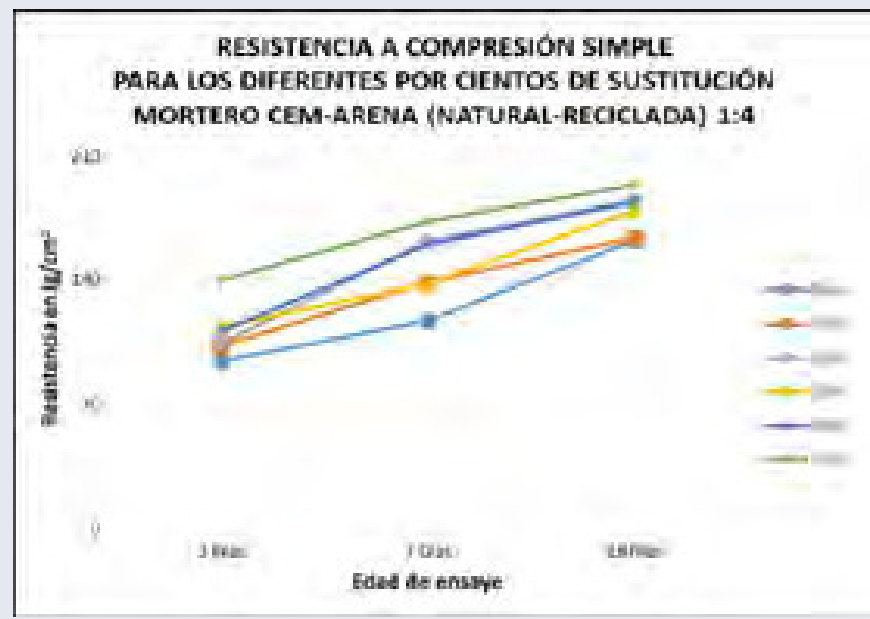

Figura 2

\section{Demanda de agua y masa unitaria 1:4}

La demanda de agua fue aumentando hasta un $8.4 \%$ conforme se aumentó el contenido de agregado reciclado y en el caso de la masa unitaria, ésta fue disminuyendo hasta un $5.6 \%$. En las figuras 3 y 4 se muestran los resultados de la demanda de agua y masa unitaria.

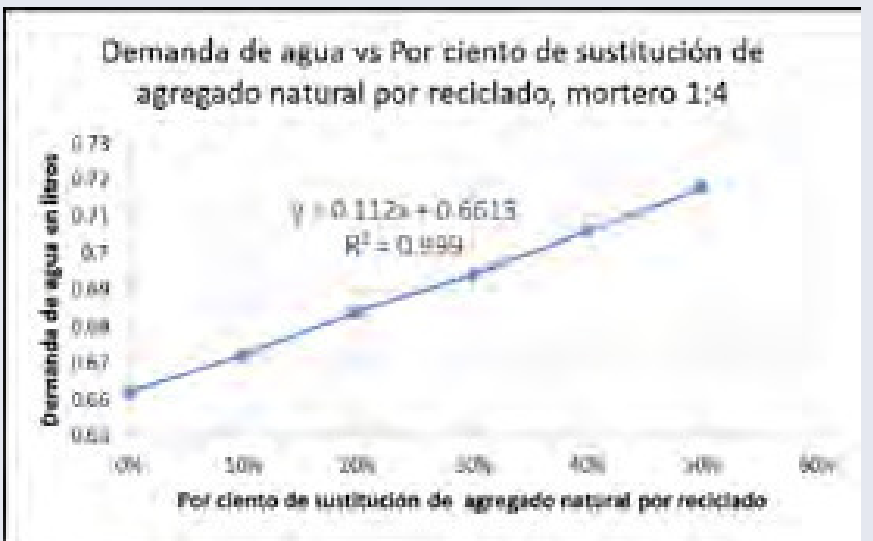

Gráfico 3
Masa unitaria vs por ciento de sustitución de agregado natural por reciclado, mortero 1:4

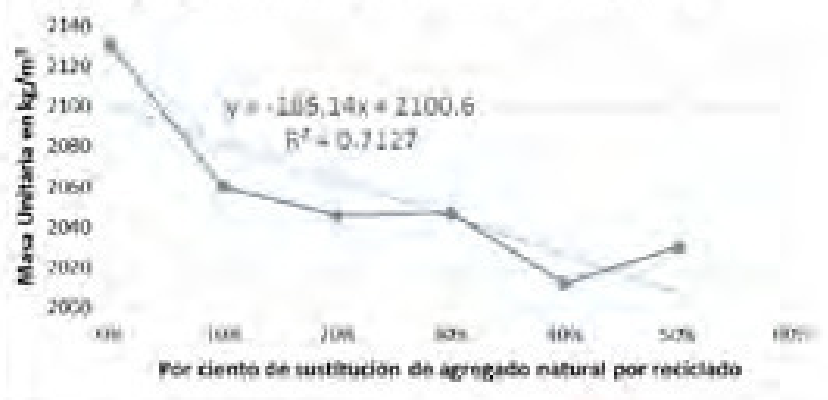

Figura 4

La figura 3, demanda de agua, se observa el modelo lineal que representa el comportamiento del incremento de agua conforme se incrementa la sustitución de agregados reciclado. El modelo lineal predice de manera adecuada dado que su valor de regresión cuadrado es casi 1 . En el caso de la masa unitaria, figura 4, el modelo lineal no predice de manera adecuada ya que su valor de regresión cuadrada es menor 0.8 , sin embargo se observa que la masa unitaria tiende a bajar pero no es significativa esta disminución.

\section{Resultados de resistencia a compresión simple 1:3}

La resistencia a compresión es mayor en el mortero elaborado con agregado reciclado para los por cientos de sustitución del $10 \%$ y $20 \%$ para las tres edades avaluadas, 7,14 y 28 días, como se observa en la figura 5. En la figura 6 se observa que el mortero con $0 \%$ se mantuvo por debajo para las diferentes edades del mortero en comparación con el $10 \%$ y $20 \%$ de sustitución.

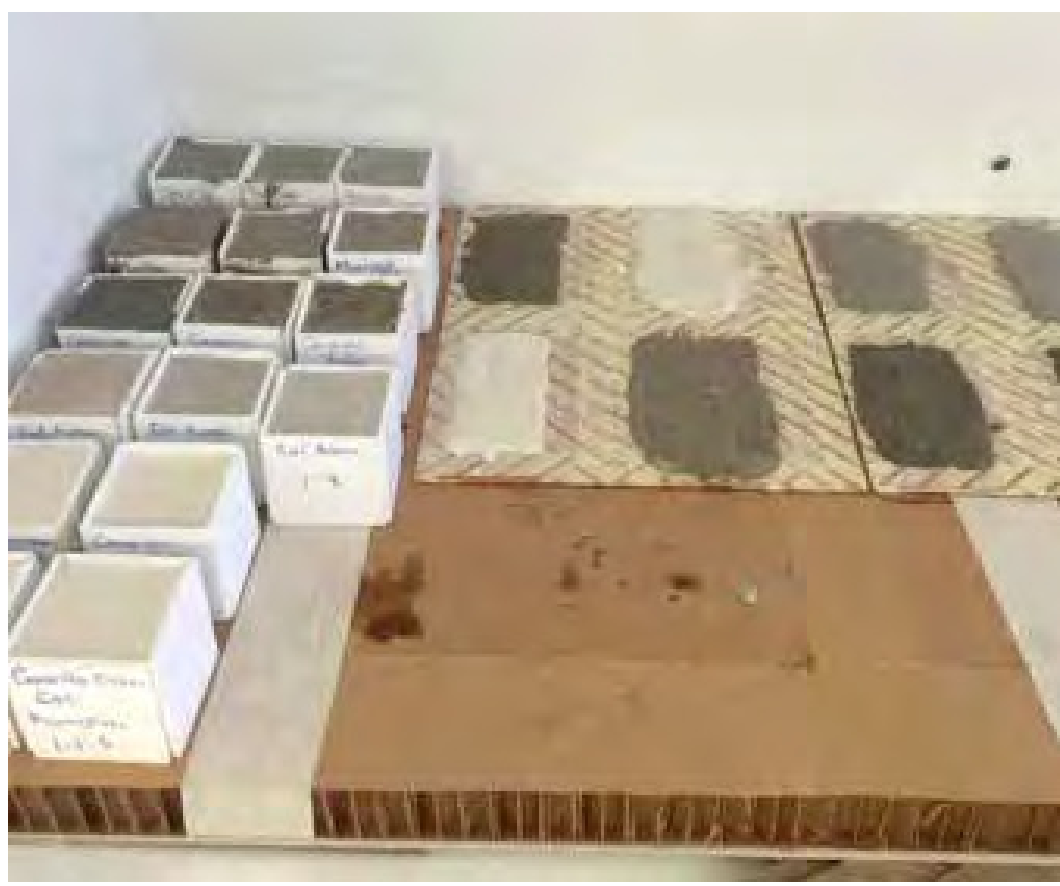




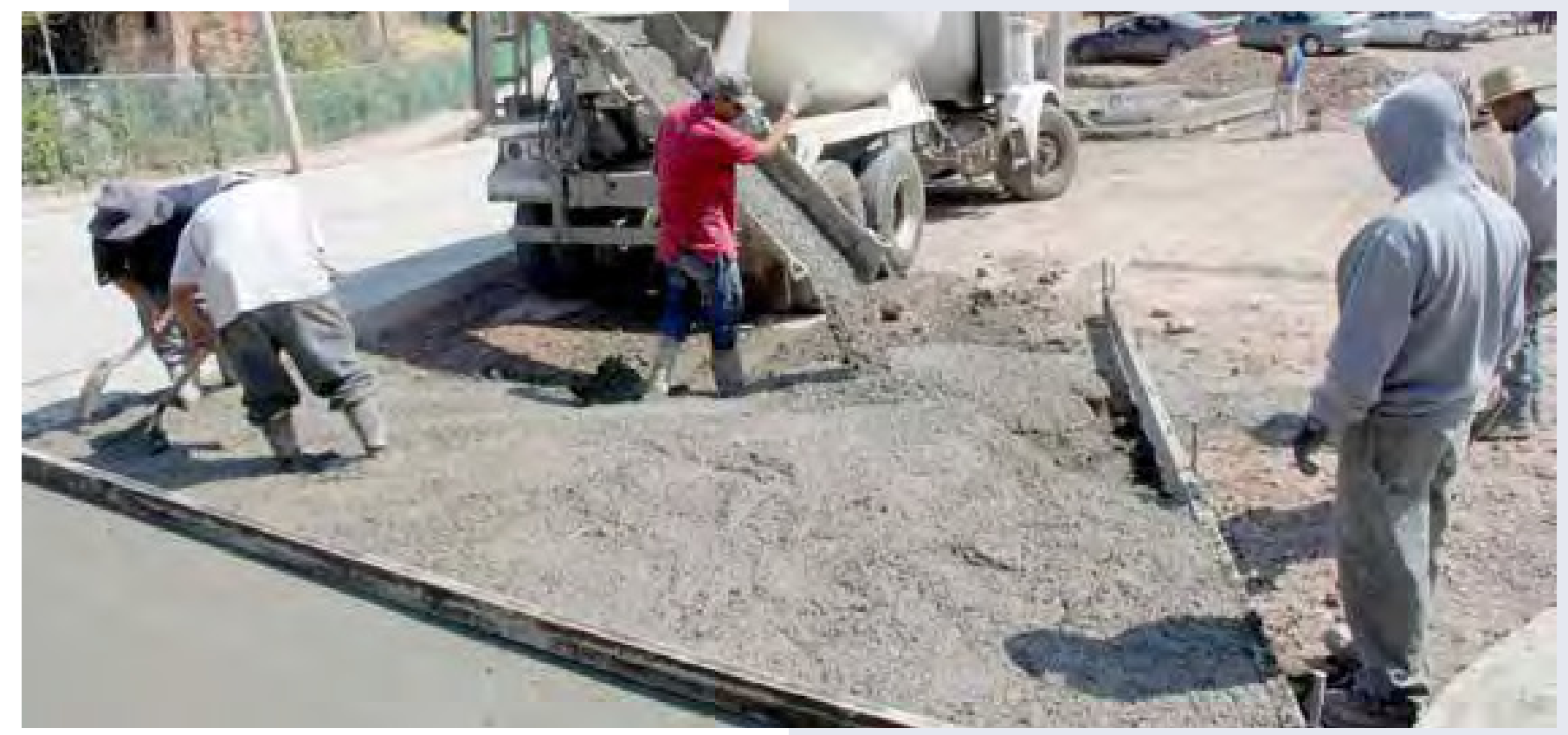

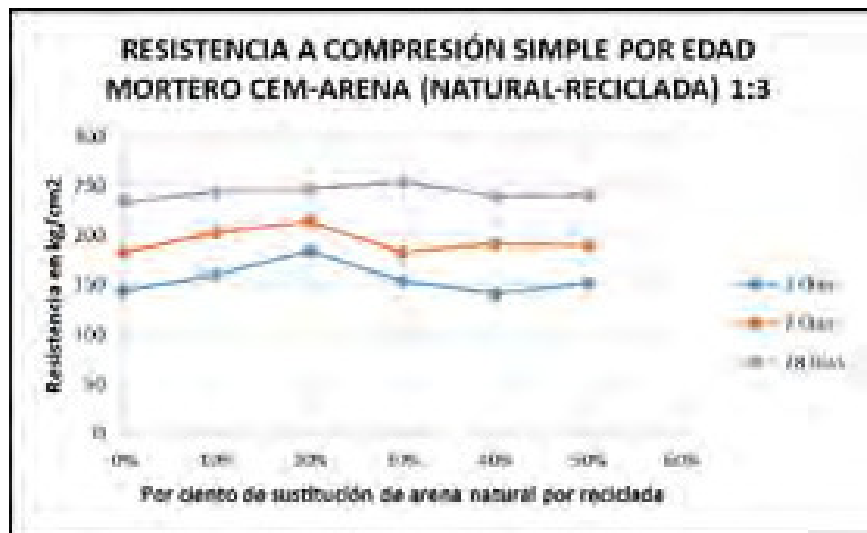

Figura 5

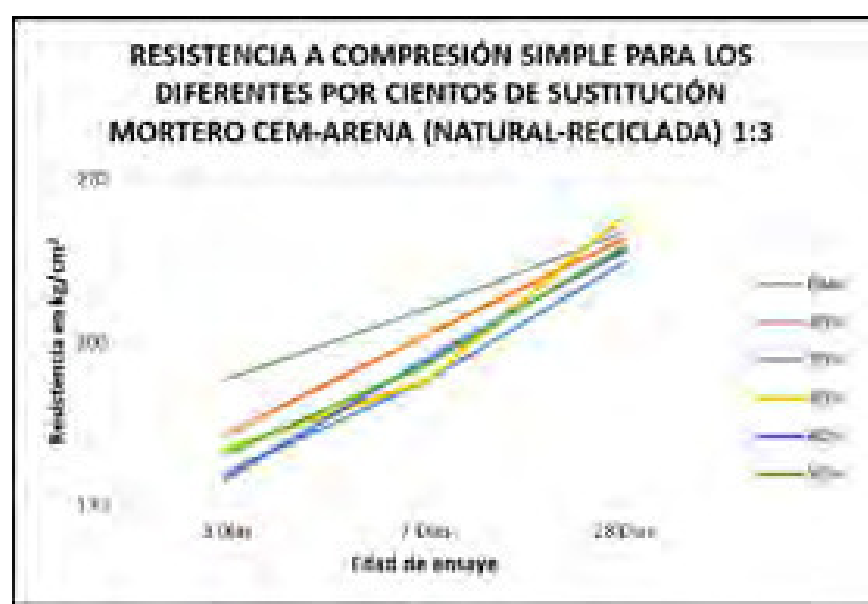

Figura 6

\section{Demanda de agua y masa unitaria 1:3}

La demanda de agua fue aumentando gradualmente pero significativamente desde un $4.2 \%$ para el $10 \%$ hasta alcanzar un $12.9 \%$ para el $50 \%$ de sustitución. En el caso de la masa unitaria, ésta fue disminuyendo en promedio el $1.3 \%$, alcanzando un valor máximo de 2.55 $\%$ para el $50 \%$ de sustitución, por lo que, tomado en cuenta los resultados podemos decir que la disminución de la masa unitaria no es significativa. En las figuras 7 y 8 se muestran los resultados de la demanda de agua y masa unitaria.

La figura 7, demanda de agua, se observa el modelo lineal que representa el comportamiento del incremento de agua conforme se incrementa la sustitución de agregados reciclado. El modelo lineal predice de manera adecuada dado que su valor de regresión cuadrado es casi 1, al igual que para el mortero 1:4. En el caso de la masa unitaria, figura 8 , el modelo lineal no predice de manera adecuada ya que su valor de $R$ cuadrada es menor 0.8 , sin embargo se observa que la masa unitaria tiende a bajar pero no es significativa esta disminución.

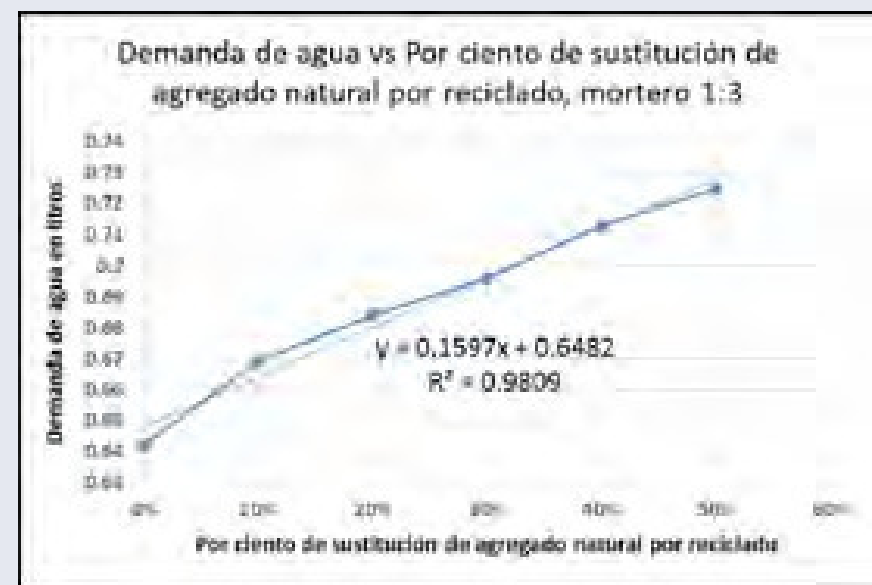

Figura 7 


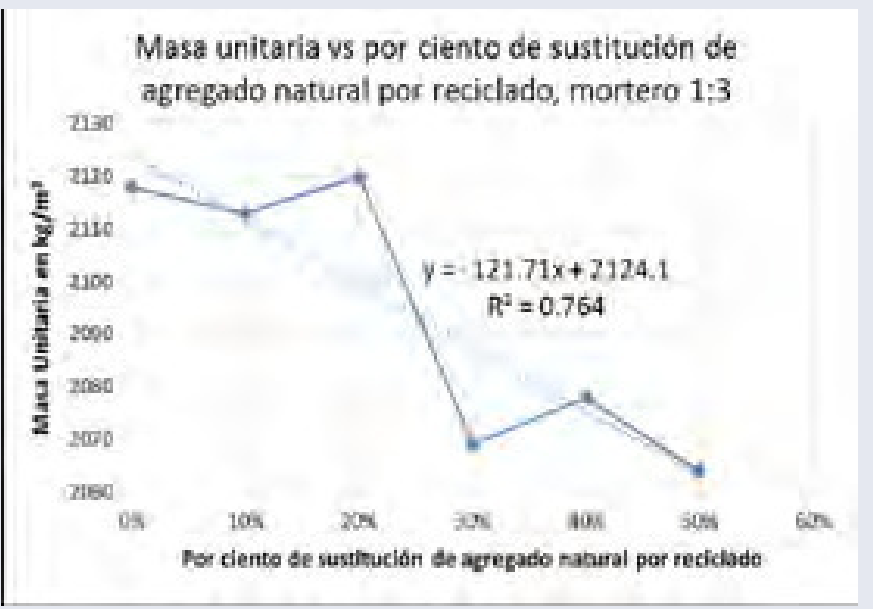

Figura 8

\section{CONCLUSIONES Y RECOMENDACIONES}

Tomando en cuenta los resultados de la resistencia a compresión simple para las dos proporciones, 1:3 y 1:4, para los diferentes por cientos de sustitución incluyendo la proporción base, para la proporción 1:3 la resistencia se incrementó para el $10 \%$ y $20 \%$ de sustitución de agregado para las tres edades evaluadas. En el caso de la proporción 1:4 la resistencia se incrementó para todos los por cientos de sustitución, alcanzando un $19.7 \%$ más resistencia la mezcla con el $50 \%$ de sustitución con respecto a la mezcla base a los 28 días.

Las propiedades en estado fresco, la masa unitaria y la fluidez, la primera disminuyo para las dos proporciones pero no de manera significativa, para las mezclas 1:3 la variación fue del orden del $1.3 \%$ en promedio con valor máximo del $2.5 \%$ y para la mezclas 1:4 fue del $3.3 \%$ hasta un valor máximo del $5.7 \%$. La demanda de agua se incrementó para las dos proporciones en sus diferentes por cientos de sustitución de agregado reciclado, éste incremento obedece al mayor por ciento de absorción que tiene el agregado reciclado con respecto al natural. Los valores obtenidos para las mezclas 1:3 la demanda fue desde el $4.7 \%$ hasta $12.9 \%$ y para las mezclas 1:4 desde un $1.5 \%$ hasta el $8.4 \%$.

Finalmente, considerando el resultado de las propiedades estudiadas para los morteros en proporción 1:3 y 1:4, es factible la utilización de la arena producto de la demolición de escombros de concreto para la fabricación de morteros con estas proporciones. Adicionalmente, el beneficio de la reutilización de desecho de concreto, es una forma de resolver el problema de la mala disposición de ellos, ya que son residuos de disposición especial y no pueden ser dispuestos en Rellenos Sanitarios Municipales, aunado a la contribución en la disminución de la explotación de bancos de materiales naturales, contribuyendo a conservar recursos naturales no renovables.

\section{BIBLIOGRAFÍA}

[1] I. Martínez y C. J. Mendoza. "Comportamiento mecánico de concreto fabricado con agregado reciclado". Revista Ingeniería, Investigación y Tecnología, Vol VII 3. 2005 Revista arbitrada y editada por el instituto de ingeniería de la UNAM. ISNN: 151-164.

[2] www.ingenieriarural.com -Universidad de Castilla La Mancha. Consultada en mayo de 2013.

[3] J.A. Domínguez y E. Martínez. "Reinserción de los residuos de construcción y demolición al ciclo de vida de la construcción de viviendas". 2007. Revista Ingeniería, Revista Académica de la FI-UADY, 11-3, pp. 43-54, ISSN: 1665-529X.

[4] M. Mamlouk. y J. Zaniewski (2009). Materiales para ingeniería civil. Madrid, 2009. Pearson Educación. Pp 167-210.

[5] A. Salazar"Un método empírico para el proporcionamiento de mezclas de morteros de Cemento Portland para albañilería". Revista Materiales de construcción Vol 35. .1985. Consejo Superior de Investigaciones Científicas España. Universidad del Valle, Cali, Colombia.

[6] Manual de ensayos de materiales, Instituto de la Construcción y Gerencia, (EM 2000), Editado por ICG. 2000.

[7] NMX-C-061-ONNCCE.- Industria de la construcciónDeterminación de la resistencia a la compresión de cementantes hidráulicos".

[8] Nadf-007-rnat-2004- Establece la clasificación y especificaciones de manejo para residuos de la construcción en el Distrito Federal.

[9] Imcyc."Proporcionamiento de mezclas". Instituto Mexicano del concreto y el Cemento, 2004. Pp-21 - 34.

[10] NMX-C-061-ONNCCE-2001 Industria de la construcción - Determinación de la resistencia a compresión de los cementos hidráulicos.

[11] NMX-C-073-ONNCCE-2004 Industria de la Construcción Agregados - Masa Volumétrica - método de prueba.

[12] NMX -C-077-ONNCCE-1997 industria de la construcción agregados para concreto - análisis granulométrico -método de prueba.

[13] NMX-C-085-ONNCCE-2002 Industria de la construcción Método estándar para el mezclado de pastas y morteros de cementantes hidráulicos.

[14] NMX-C-164-ONNCCE-2004 Industria de la Construcción - Agregados - Determinación de la Masa Específica y Absorción de Agua del agregado grueso.

[15] NMX-C-165-ONNCCE-2004 Industria de la Construcción - Agregados - Masa Específica y Absorción de Agua del agregado fino - método de prueba.

[16] NMX-C-196-ONNCCE-2010 Industria de la construcción Resistencia a ala abrasión para agregado grueso, desgaste de los ángeles - método de prueba

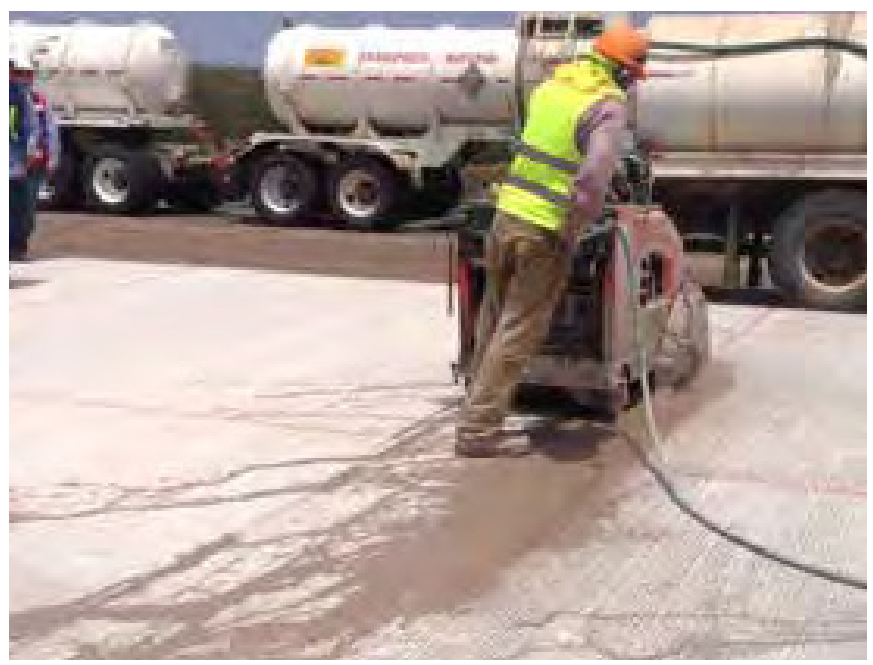

